\title{
Biomarker triplet NAMPT/VEGF/HER2 as a de novo detection panel for the diagnosis and prognosis of human breast cancer
}

\author{
YANYAN ZHU ${ }^{1}$, MEIYAN GUO ${ }^{2}$, LINGYUN ZHANG ${ }^{3,4}$, TAO XU $^{5}$, LI WANG ${ }^{6}$ and GUOXIONG XU 3 \\ ${ }^{1}$ Department of General Surgery, Jinshan Hospital, Fudan University, Shanghai 201508; ${ }^{2}$ Affiliated Hospital \\ of Hebei University of Engineering, Handan, Hebei 056002; ${ }^{3}$ Center Laboratory, Jinshan Hospital, \\ Fudan University, Shanghai 201508; ${ }^{4}$ Department of Oncology, Shanghai Medical College, \\ Fudan University, Shanghai 200032; ${ }^{5}$ Health Check-Up Center, ${ }^{6}$ Department of Pathology, \\ Jinshan Hospital, Fudan University, Shanghai 201508, P.R. China
}

Received July 27, 2015; Accepted September 23, 2015

DOI: $10.3892 /$ or.2015.4391

\begin{abstract}
The early detection of breastcancer, the mostcommon malignant tumor disease in women worldwide, relies on mammography and self breast examination. Here we evaluated the concentration of nicotinamide phosphoribosyltransferase (NAMPT), vascular endothelial growth factor (VEGF) and human epidermal growth factor receptor-2 (HER2) in serum and their expression in breast tissues associated with the clinicopathological features of patients with benign and malignant breast tumors. The immunohistochemical analysis showed that NAMPT, VEGF and HER2 proteins were overexpressed in breast tumors. The highest expression was observed in malignant tumors, low in benign tumors and negative in the adjacent normal tissue, indicating that the triplets may be progression markers and correlated with each other. The detection rate of NAMPT, VEGF and HER2 alone in tissue was 54.17, 64.58 and $60.42 \%$, respectively, and was increased to about $79 \%$ in double combination and to $90 \%$ in triple combination. The basal levels of serum NAMPT, VEGF and HER2 in healthy controls were $94.90 \pm 4.24 \mathrm{pg} / \mathrm{ml}, 87.02 \pm 2.41 \mathrm{pg} / \mathrm{ml}$ and $1.12 \pm 0.04 \mathrm{ng} / \mathrm{ml}$, respectively, measured by ELISA and found to be increased by 6.64-, 1.76- and 2.52-fold, respectively, in patients with malignant breast tumor. These elevated serum
\end{abstract}

Correspondence to: Professor Guoxiong Xu, Center Laboratory, Jinshan Hospital, Fudan University, 1508 Longhang Road, Shanghai 201508, P.R. China

E-mail: guoxiong.xu@fudan.edu.cn

Abbreviations: HER2, human epidermal growth factor receptor-2; ELISA, enzyme-linked immunosorbent assay; IHC, immunohistochemistry; M-AO, malignant tumor afteroperation; $\mathrm{M}-\mathrm{BO}$, malignant tumor before operation; NAMPT, nicotinamide phosphoribosyltransferase; SD, standard deviation; SEM, standard error of mean; SI, staining index; VEGF, vascular endothelial growth factor; WHO, World Health Organization

Key words: breast cancer, tumor biomarker, diagnosis, tumor progression, early detection, serum panel levels of NAMPT, VEGF and HER2 in patients were decreased after tumor removal, suggesting that these molecules are the indicators of treatment efficacy. The combined measurement of these triplets together may improve the sensitivity of breast cancer diagnosis and may potentially be used as a testing panel for the detection of malignant tumors, the assessment of treatment effectiveness and the monitoring of the disease progression in patients with breast cancer. Thus, we propose that the biomarker triplet NAMPT/VEGF/HER2 can be used as a de novo detection panel for the diagnosis and prognosis of human breast cancer.

\section{Introduction}

Breast cancer is the most common malignant tumor disease in women. Around 1.38 million newly diagnosed cancer occur worldwide on an annual basis and among these, breast cancer accounts for $23 \%$ of all cases (1). The World Health Organization (WHO) estimates that about half of breast cancer cases with $60 \%$ mortality are found in developing countries. In China, the incidence and mortality of breast cancer were increased from 169,452 and 44,908 in 2008 to 187,000 and 48,000 in 2012 , respectively (GLOBOCAN 2012) (http://globocan.iarc.fr/). Currently, the early detection of breast cancer relies on mammography and self breast examination. Although several potential serum biomarkers of breast cancer have been proposed, the clinical significance using quantitative methods has barely been validated.

Nicotinamide phosphoribosyltransferase (NAMPT), also known as visfatin or pre-B cell enhancing factor (PBEF), is an insulin-mimetic adipocytokine highly expressed in and secreted by visceral adipose tissue associated with obesity $(2,3)$. The overexpression of NAMPT has been found in the different types of human malignant tumors, including colorectal, gastric, endometrial, ovarian, breast, prostate and thyroid cancers, myeloma, melanoma, astrocytomas/glioblastoma and other carcinomas (4-13). Plasma NAMPT levels in patients with breast cancer are higher than in healthy controls (14). Vascular endothelial growth factor (VEGF), an 
angiogenic factor expressed in the endothelial cells of blood vessels, plays a role in the process of tumor angiogenesis. Many studies showed that circulating VEGF is elevated in cancer patients. The high concentration of VEGF and its soluble receptor in the serum of patients with breast cancer are held responsible for the disease as they show positive correlations with the clinical stages (15). Elevated level of VEGF has been shown in early breast cancer patients compared with healthy controls (16). Human epidermal growth factor receptor-2 (HER2), an oncogene also known as Her-2/neu or $c-e r b B-2$, has been reported to have significantly high serum levels in breast cancer patients compared with healthy controls and in metastatic breast cancer patients compared with the non-metastatic ones (17). Previous report also showed that no correlation was found between preoperative and perioperative serum VEGF and HER2 in patients with early breast cancer (18). Thus, it is speculated that NAMPT, VEGF and HER2 are three variables that may collectively be useful as early diagnostic markers of breast cancer. However, whether the absolute change of NAMPT is correlated with VEGF and HER2 in benign and malignant breast tumors is unknown. Furthermore, whether the circulating NAMPT/VEGF/HER2 triplets are associated with the clinicopathological characteristics in patients with breast cancer is not yet explored.

The present study was performed to examine the expression of NAMPT, VEGF and HER2 in benign and malignant breast tumors and to investigate whether the expression of NAMPT, VEGF and HER2 is associated with the clinicopathological features of human breast cancer. Moreover, we evaluated the serum levels of NAMPT, VEGF and HER2 in breast cancer patients before and after tumor removal. Finally, we analyzed the association of serum levels of these biomarkers with their expression in the breast tissues of cancer patients.

\section{Materials and methods}

Patients and tissue preparation. The study on human subjects was approved by the Ethics Committee of Jinshan Hospital, Fudan University, Shanghai, China. Samples from patients who were primarily diagnosed with breast tumor at Jinshan Hospital from 2013 to 2014 were retrieved for the present study. None of the patients had received radiotherapy or chemotherapy before surgery. A total of 68 paraffin-embedded samples constituting 20 benign tumors and 48 malignant tumors were subjected to the histopathological examination and immunohistochemistry. The adjacent normal tissues were used as controls. The $10 \%$ formalin-fixed paraffin-embedded breast tissue specimens were prepared. Four micrometer thick sections of these specimens were stained by hematoxylin and eosin (H\&E) to confirm the histological characteristics. The histological grades and clinical stages of tumor were classified by experienced surgeons and pathologists based on the WHO classification.

Immunohistochemical staining and analysis. To evaluate the expression of NAMPT, VEGF and HER2 proteins in breast tumors, immunohistochemical staining was performed as described previously (19). Briefly, after blocking, the sections were incubated with a rabbit monoclonal anti-NAMPT, mouse monoclonal anti-VEGF or anti-HER2 antibody (all 1:250 dilution; Abcam, Cambridge, MA, USA) at $4{ }^{\circ} \mathrm{C}$ overnight, followed by incubation with biotinylated secondary antibody (1:150 dilution; Maixin Bio, Fuzhou, China) at room temperature for $1 \mathrm{~h}$. After washing, the signal was detected using a DAB kit (diaminobenzidine; Maixin Bio). Finally, the sections were counterstained with hematoxylin and photographed under a light microscope (BX43; Olympus, Tokyo, Japan).

Double blind scoring of NAMPT, VEGF and HER2 immunoreactive staining was performed independently by two examiners who had no prior knowledge of patient's clinical status. The sections were evaluated at original magnification, $x 200$. The proportion of cells exhibiting protein expression was scored by the extent of immunoreactive staining and was assigned to one of the following categories as described previously (19). Briefly, the percentage of positive cells was scored as follows: no reactivity as $0, \leq 25 \%$ positive cells as $1,26-50 \%$ positive cells as $2,51-75 \%$ positive cells as 3 and $>75 \%$ positive cells as 4 . The intensity of staining was scored as follows: no staining as 0 , weak staining as 1 , moderate staining as 2 , and strong staining as 3 . The final staining index (SI) was developed based on the sum score of the positive staining and intensity. The SI score was then clustered into four groups: $0, \leq 2$ sum points; $1,3-4$ sum points; 2, 5-6 sum points; 3, 7 sum points. Finally, the cases were categorized based on the SI score $0-1$ to be negative and 2-3 to be positive.

Blood sample collection. Blood samples were collected from 30 patients with a malignant tumor on a day before operation (M-BO) and the 7th day after operation (M-AO) as well as from 28 patients with benign tumor. For comparison, 30 blood samples from age- and body mass index (BMI)-matched healthy controls were obtained from the Health Check-Up Center of Jinshan Hospital in 2014. The median age of healthy controls and patients with malignant tumor was 50.00 and 52.00, respectively, and the median BMI of healthy controls and patients with malignant tumor were 23.05 and 23.73, respectively. All subjects (patients and healthy controls) who enrolled into the study signed a consent form prior to the collection of blood samples. Serum was prepared using a serum separator tube (SST) which allowed samples to clot for $30 \mathrm{~min}$ before centrifugation for $15 \mathrm{~min}$ at $1,000 \mathrm{x} \mathrm{g}$, aliquoted, and stored at $-80^{\circ} \mathrm{C}$ until use. None of the samples were previously thawed.

Enzyme-linked immunosorbent assay. Serum levels of NAMPT, VEGF and HER2 were determined in patients and healthy individuals by enzyme-linked immunosorbent assay (ELISA). NAMPT and HER2 kits were purchased from Wuhan Xinqidi Biological Technology Co., Ltd. (Wuhan, Hubei, China), whereas VEGF kit was purchased from R\&D Systems (Human VEGF Immunoassay, Quantikine ${ }^{\circledR}$ ELISA; R\&D Systems, Inc., Minneapolis, MN, USA). Briefly, after adding $100 \mu \mathrm{l}$ assay diluent into each well, $100 \mu \mathrm{l}$ standards and serum samples were, respectively, added into the wells and incubated at room temperature for $2 \mathrm{~h}$. After washing away any unbound substances, $200 \mu 1$ enzyme-linked polyclonal antibody specific to NAMPT, VEGF, or HER2 was added into each well and incubated at room temperature for $2 \mathrm{~h}$. After washing 3 times, $200 \mu \mathrm{l}$ substrate solution was added into 

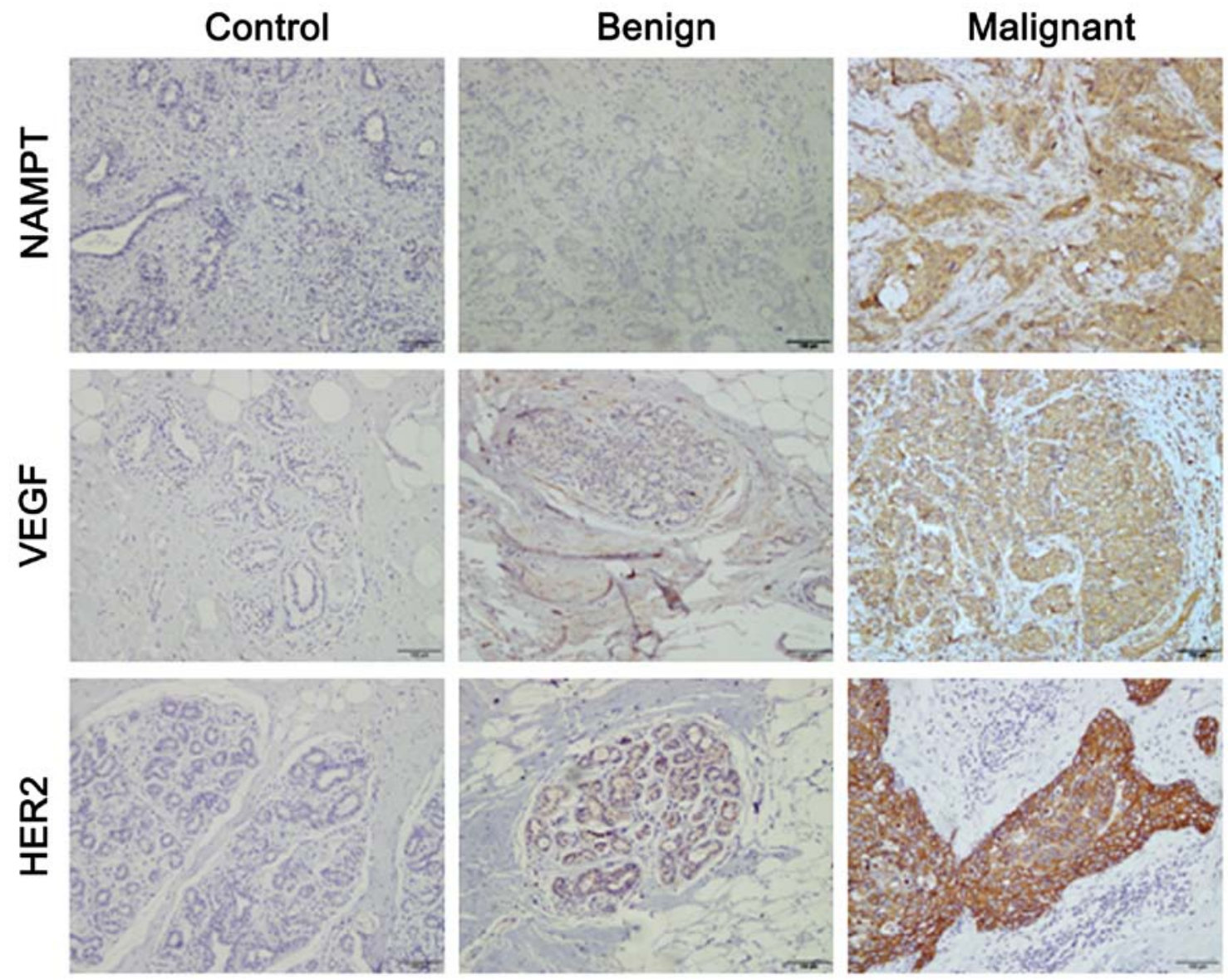

Figure 1. Immunohistochemical staining of NAMPT, VEGF and HER2 in breast tissues. Representative images are shown. A brown color in the cytoplasm is considered as positive staining. Control, adjacent normal breast tissue; benign, benign tumor; malignant, malignant tumor. Original amplification, $\mathrm{x} 200$. Scale bar, $100 \mu \mathrm{m}$.

each well and incubated at room temperature for $25 \mathrm{~min}$. After adding $50 \mu \mathrm{l}$ stop solution into each well, the optical density of each well was determined within 30 min using a microplate reader at $450 \mathrm{~nm}$.

Statistical analysis. Statistical analyses were performed with SPSS Statistics 21.0 software (SPSS, Chicago, IL, USA). Based on the SI system, the categories on positivity and negativity were classified. Statistical evaluation was performed using $\chi^{2}$ test to analyze the association between the expression of NAMPT/VEGF/HER2 and the clinicopathological characteristics and to compare the positivity between benign and malignant tumors. For comparison of each variable in breast malignant tumors, a McNemar test was performed. For multiple group comparison, an ANOVA was applied. Significant difference between 2 groups was analyzed by a Student's t-test. For testing correlation between different serum markers, a linear regression was applied. Data are presented as the mean \pm standard deviation (SD) or standard error of mean (SEM) as indicated. $\mathrm{P}<0.05$ was considered to indicate a statistically significant difference.

\section{Results}

NAMPT, VEGF and HER2 as breast tumor progression markers. The expression of NAMPT, VEGF and HER2 in benign and malignant breast tumors was detected by immunohistochemical staining. The adjacent normal breast tissue was used as normal control. We found that the expression of NAMPT, VEGF and HER2 was undetectable or barely detectable in controls, whereas the aberrant expression of NAMPT, VEGF and HER2 was noted in human benign and malignant breast tumors (Fig. 1). By comparison of all breast tissues, the highest degree of the expression of NAMPT, VEGF and HER2 was observed in malignant tumors. After the assessment of the SI score as indicated above, we classified the expression level into positive and negative categories. Compared with benign tumors, the positive rate of NAMPT, VEGF and HER2 expression was significantly increased in malignant tumors (all $\mathrm{P}<0.01)$ (Table I). These data suggest that NAMPT, VEGF and HER2 may be the indicators or progression markers of breast cancer development.

Association of NAMPT, VEGF and HER2 expression with the clinicopathological characteristics of breast cancer. To examine the association of the expression of NAMPT, VEGF, and HER2 with the clinicopathological characteristics of breast cancer, a Chi-square test was applied. All patient information was gathered by reviewing medical charts and the records of pathology. By comparison of 48 malignant tumors, we found that the expression of NAMPT, VEGF and 
Table I. Expression of NAMPT, VEGF and HER2 in breast benign and malignant tumors.

\begin{tabular}{|c|c|c|c|c|c|c|c|}
\hline \multirow[b]{2}{*}{ Tumor } & \multirow[b]{2}{*}{$\mathrm{n}$} & \multicolumn{2}{|c|}{ NAMPT } & \multicolumn{2}{|c|}{ VEGF } & \multicolumn{2}{|c|}{ HER 2} \\
\hline & & $\begin{array}{c}\text { Positive } \\
\mathrm{n}(\%)\end{array}$ & $\begin{array}{c}\text { Negative } \\
\text { n }(\%)\end{array}$ & $\begin{array}{c}\text { Positive } \\
\text { n }(\%)\end{array}$ & $\begin{array}{c}\text { Negative } \\
\mathrm{n}(\%)\end{array}$ & $\begin{array}{c}\text { Positive } \\
\text { n }(\%)\end{array}$ & $\begin{array}{c}\text { Negative } \\
\text { n }(\%)\end{array}$ \\
\hline Benign & 20 & $2(10.0)$ & $18(90.0)$ & $3(15.0)$ & $17(85.0)$ & $5(25.0)$ & $15(75.0)$ \\
\hline Malignant & 48 & $26(54.2)$ & $22(45.8)$ & $31(64.6)$ & $17(35.4)$ & $29(60.4)$ & $19(39.6)$ \\
\hline P-value & & \multicolumn{2}{|c|}{0.001} & \multicolumn{2}{|c|}{$<0.001$} & \multicolumn{2}{|c|}{0.008} \\
\hline
\end{tabular}

For comparison of benign and malignant tumors, a $\chi^{2}$ test was applied. The expression of NAMPT, VEGF and HER 2 was significantly different between benign and malignant tumors. $n$, number of cases.

Table II. Association of the expression of NAMPT, VEGF,and HER2 proteins with the clinicopathological features of patients with breast cancer.

\begin{tabular}{llllll}
\hline $\begin{array}{l}\text { Clinicopathological } \\
\text { features }\end{array}$ & & NAMPT & & VEGF & HER2 \\
\cline { 1 - 2 } & & + & + & + & - \\
\hline
\end{tabular}

\section{Age (years)}

at diagnosis

$\begin{array}{lccccccc}\leq 50 & 23 & 13 & 10 & 15 & 8 & 14 & 9 \\ >50 & 25 & 13 & 12 & 16 & 9 & 15 & 10 \\ \text { P-value } & & 0.753 & 0.930 & 0.951\end{array}$

Tumor size

$\begin{array}{lccccccc}\leq 2 \mathrm{~cm} & 19 & 10 & 9 & 11 & 8 & 12 & 7 \\ >2 \mathrm{~cm} & 29 & 16 & 13 & 20 & 9 & 17 & 12 \\ \text { P-value } & & 0.863 & 0.433 & 0.753 \\ \text { LN metastasis } & & & & & & & \\ \text { Yes } & 17 & 11 & 6 & 14 & 3 & 8 & 9 \\ \text { No } & 31 & 15 & 16 & 17 & 14 & 21 & 10 \\ \text { P-value } & & 0.278 & 0.057 & 0.161\end{array}$

Clinical stage

$\begin{array}{lrrrrrrc}\text { 0-II } & 38 & 18 & 20 & 15 & 23 & 25 & 13 \\ \text { III-IV } & 10 & 8 & 2 & 2 & 8 & 4 & 6 \\ \text { P-value } & & & 0.137^{\mathrm{a}} & 0.439^{\mathrm{a}} & 0.263^{\mathrm{a}}\end{array}$

The expression of NAMPT, VEGF, and HER2 proteins was detected by immunohistochemistry. For comparison of NAMPT, VEGF and HER2 expression associated with the clinicopathological features, the $\chi^{2}$ test was applied. $\mathrm{n}$, number of cases; + , positive expression; -, negative expression; LN, lymph node. ${ }^{\mathrm{a}} \mathrm{Chi}$-square test with continuity correction.

HER2 was not significantly associated with age ( $\leq 50 \mathrm{vs.}>50)$, tumor size ( $\leq 2$ vs. $>2 \mathrm{~cm}$ ), lymph node metastasis (yes vs. no) and clinical stage (0-II vs. III-IV) in patients with breast cancer (all $\mathrm{P}>0.05$ ) (Table II).

Correlation of the expression between NAMPT, VEGF and HER2 in breast cancer. Next, we compared these triple variables with each other to see whether the overexpression
Table III. Correlation between NAMPT and VEGF expression, Nampt and HER2 expression, and VEGF and HER2 expression in breast malignant tumors.

\begin{tabular}{|c|c|c|c|c|}
\hline & $\begin{array}{c}\text { Positive } \\
\text { (n) }\end{array}$ & $\begin{array}{l}\text { Negative } \\
\text { (n) }\end{array}$ & Total & P-value \\
\hline & \multicolumn{2}{|c|}{ VEGF } & & \\
\hline \multicolumn{5}{|l|}{ NAMPT } \\
\hline Positive & 24 & 2 & 26 & \\
\hline Negative & 7 & 15 & 22 & \\
\hline \multirow[t]{2}{*}{ Total } & 31 & 17 & 48 & $\mathrm{P}=0.180$ \\
\hline & \multicolumn{2}{|c|}{ HER2 } & & \\
\hline \multicolumn{5}{|l|}{ NAMPT } \\
\hline Positive & 16 & 10 & 26 & \\
\hline Negative & 13 & 9 & 22 & \\
\hline \multirow[t]{2}{*}{ Total } & 29 & 19 & 48 & $\mathrm{P}=0.678$ \\
\hline & \multicolumn{2}{|c|}{ HER2 } & & \\
\hline \multicolumn{5}{|l|}{ VEGF } \\
\hline Positive & 18 & 13 & 31 & \\
\hline Negative & 11 & 6 & 17 & \\
\hline Total & 29 & 19 & 48 & $\mathrm{P}=0.839$ \\
\hline
\end{tabular}

For comparison of the expression of NAMPT vs. VEGF, NAMPT vs. HER2, and VEGF vs. HER2, a McNemar test was performed. There was no significant difference among the comparisons. $n$, number of cases.

of NMAPT, VEGF and HER2 in breast malignant tumor is correlated. Using a McNemar test, we found that there was no significant difference among the comparisons, such as NAMPT vs. VEGF, VEGF vs. HER2 and HER2 vs. NAMPT (all P>0.05) (Table III). These data in turn suggest that the expression of NAMPT, VEGF and HER2 may be correlated. Further analysis showed that the detection rate of NAMPT, VEGF and HER 2 in combination was increased in breast cancer patients. The detection rate of NAMPT, VEGF and HER2 alone was 54.17, 64.58 and 60.42 , respectively. The detection rate of NMAPT plus VEGF, VEGF plus HER2, and HER2 plus NAMPT was 

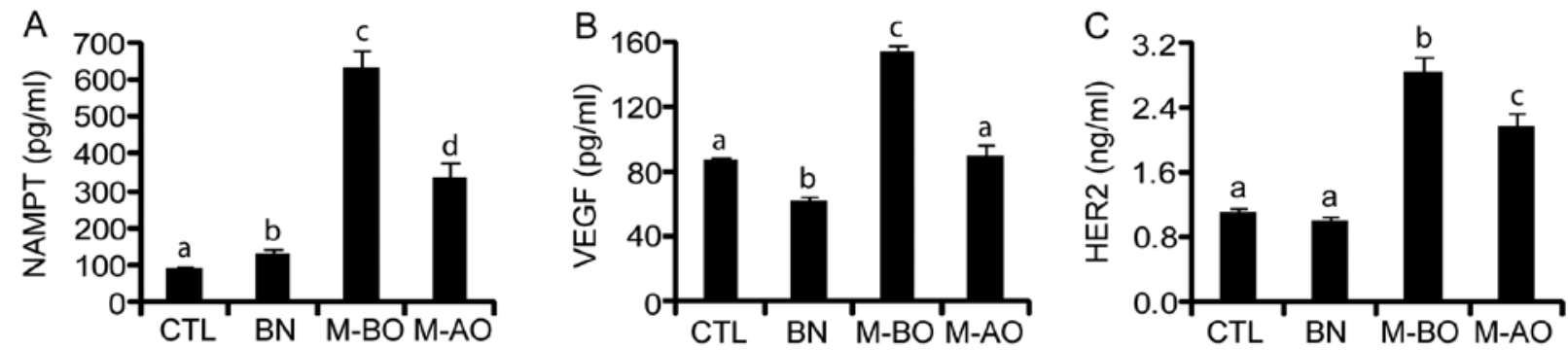

Figure 2. Concentration of serum NAMPT, VEGF and HER2. The concentrations of serum NAMPT (A), VEGF (B) and HER2 (C) in healthy controls $(\mathrm{CTL} ; \mathrm{n}=30)$, patients with a benign tumor $(\mathrm{BN} ; \mathrm{n}=28)$ and a malignant tumor before $(\mathrm{M}-\mathrm{BO})$ and after $(\mathrm{M}-\mathrm{AO})$ operation $(\mathrm{n}=30)$ were measure by ELISA Data are presented as the mean \pm SEM. Different superscript denotes statistically significant difference $(\mathrm{P}<0.05)$.
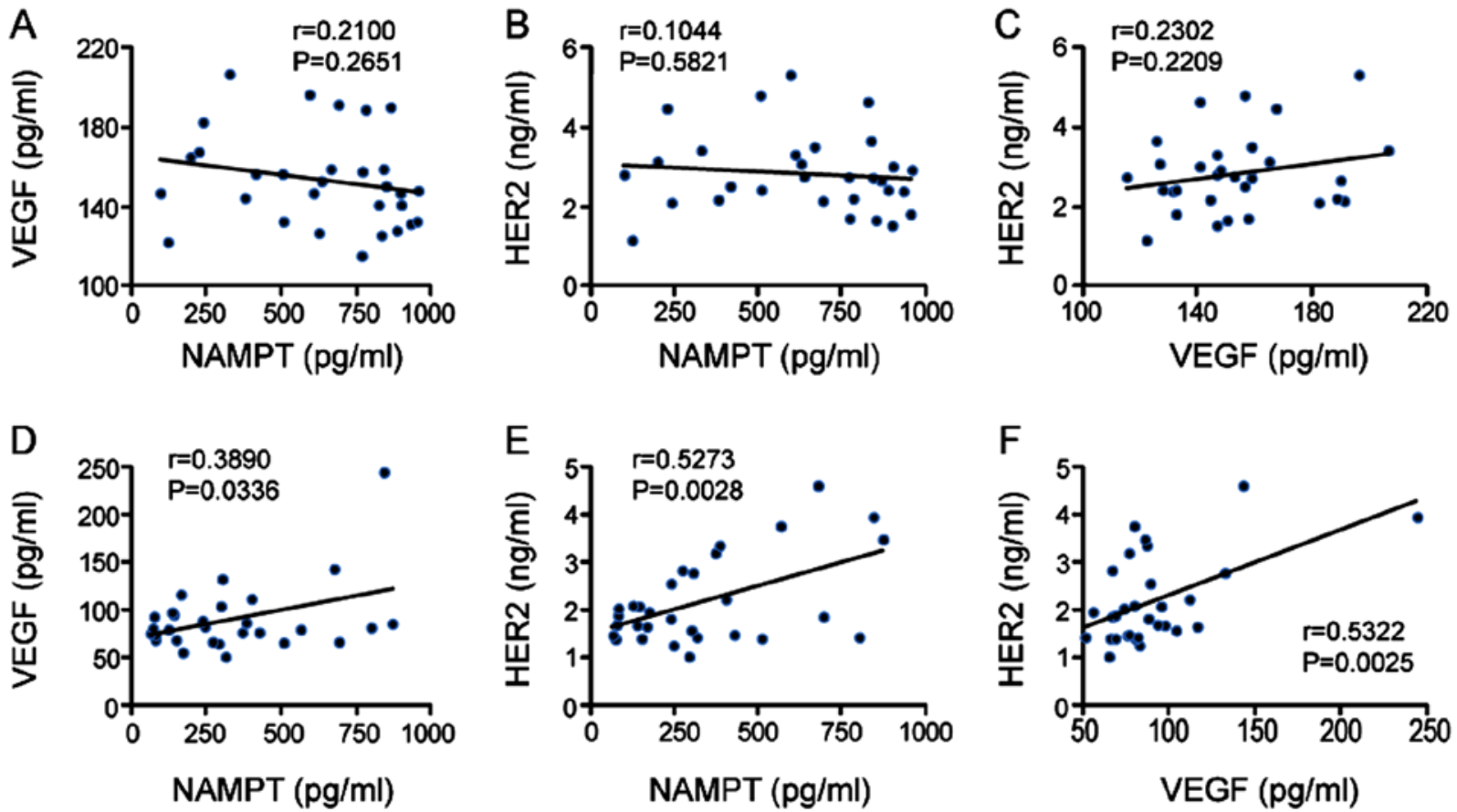

Figure 3. Correlation of NAMPT, VEGF and HER2 in serum. The concentrations of serum NAMPT, VEGF and HER2 were measure by ELISA. The correlation between the variables was examined by a linear regression before operation (A-C) and after operation (D-F). (A and D) VEGF vs. NAMPT; (B and E) NAMPT vs. HER2; (C and F) HER2 vs. VEGF.

increased to $68.75,87.50$ and $81.25 \%$, respectively. Finally, the detection rate of collective triplets reached $89.58 \%$.

Evaluation of the serum concentrations of NAMPT, VEGF and HER2 in healthy women and patients with breast cancer. Since NAMPT, VEGF and HER2 are secretory proteins, next we examined their serum levels using ELISA and compared the difference of their concentrations in healthy controls and patients with benign and malignant breast tumors before and after tumor removal. Two factors, i.e., age and BMI, were considered when comparisons were performed, but no significant difference was found between healthy controls and breast cancer patients, indicating that their age and BMI were matched between the two groups. The basal levels of serum NAMPT, VEGF and HER2 in healthy controls (CTL; $n=30$ ) were $94.90 \pm 4.24 \mathrm{pg} / \mathrm{ml}, 87.02 \pm 2.41 \mathrm{pg} / \mathrm{ml}$ and $1.12 \pm 0.04 \mathrm{ng} / \mathrm{ml}$, respectively. The concentrations of serum NAMPT, VEGF and HER 2 in patients with benign tumor $(\mathrm{BN} ; \mathrm{n}=28)$ presented different trends. In patients with a benign tumor, serum
NAMPT was slightly increased $(\mathrm{P}<0.05)$ (Fig. 2A), whereas serum VEGF was slightly decreased $(\mathrm{P}<0.01)$ (Fig. 2B), compared with healthy controls. However, no significant difference of serum HER2 was observed between healthy controls and patients with a benign tumor ( $\mathrm{P}>0.05)$ (Fig. 2C). In patients with a malignant tumor before operation (M-BO, $\mathrm{n}=30$ ), the serum levels of all three variables were significantly elevated (all $\mathrm{P}<0.001)$ and there were 6.64-, 1.76-, and 2.52-fold increases of the concentration of NAMPT, VEGF and HER2, respectively. Interestingly after the operation (M-AO), the elevated serum NAMPT, VEGF, and HER2 levels significantly declined 47, 41 and 23\%, respectively (all $\mathrm{P}<0.05$ ), and that of VEGF almost returned to the basal level of control (Fig. 2B).

Correlation of serum levels of NAMPT, VEGF and HER2 with each other in breast cancer patients. We further examined whether serum levels of NAMPT, VEGF and HER 2 are correlated with each other in patients with malignant tumors. Before operation, no correlation was observed between them 

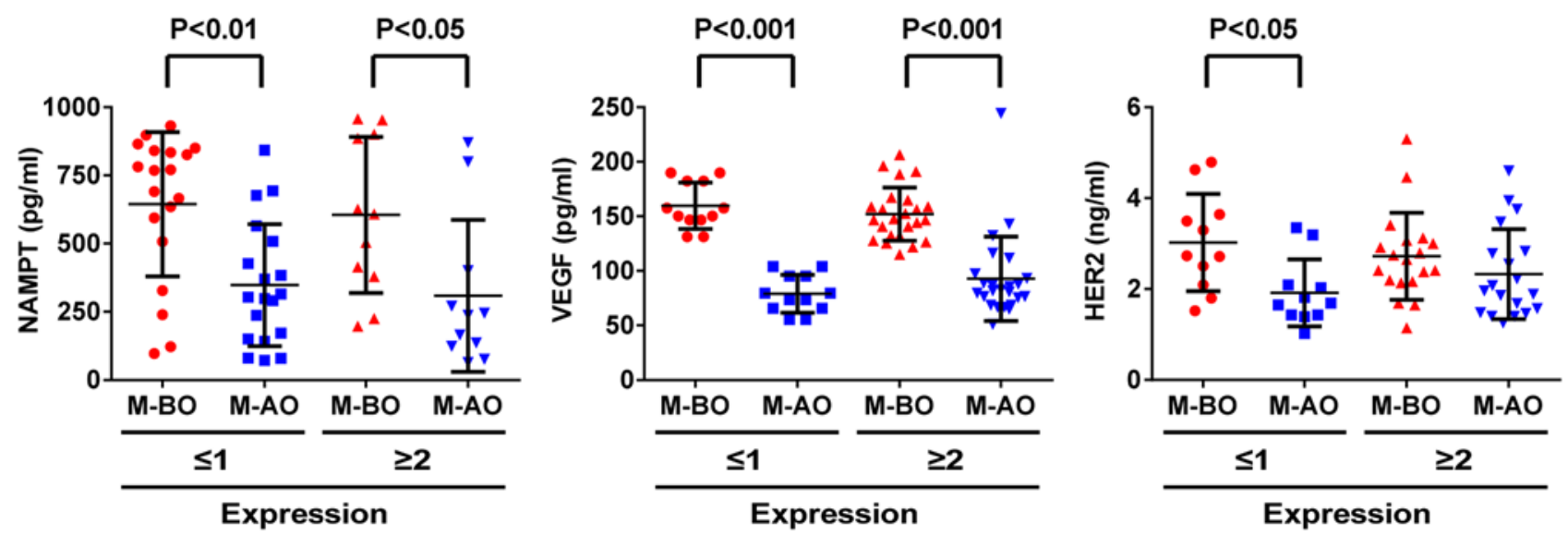

Figure 4. Association of serum concentration of NAMPT, VEGF and HER2 with their expression in patients with a malignant tumor before and after operation. The serum levels of NAMPT, VEGF and HER2 were measured by ELISA before operation (M-BO, red) and after operation (M-AO, blue). The expression of NAMPT, VEGF and HER2 in malignant tumor was detected by immunohistochemistry. The expression of NAMPT, VEGF and HER2 was assigned as negative (SI score $\leq 1$ ) or positive (SI score $\geq 2$ ). Dot is presented as an individual measure. Data are presented as the mean \pm SD.

(VEGF vs. NAMPT, NAMPT vs. HER2 and HER2 vs. VEGF; all $\mathrm{P}>0.05$ ) (Fig. 3A-C); however after tumor removal, a significant correlation was found: VEGF vs. NAMPT $(\mathrm{P}=0.0336)$ (Fig. 3D), NAMPT vs. HER2 (P=0.0028) (Fig. 3E) and HER2 vs. VEGF ( $\mathrm{P}=0.0025)$ (Fig. 3F).

Association of serum concentration of NAMPT, VEGF and HER2 with their tissue expression in patients with a malignant tumor before and after operation. Since the presence and absence of a tumor seemed to affect the serum levels of NAMPT, VEGF and HER2, next we examined the association between serum levels and tissue expression of these variables in patients with a malignant tumor before and after operation. We found that the serum levels of NAMPT and VEGF were higher in patients before operation than those after operation and that serum levels were not associated with the positivity (SI score $\geq 2$ ) and negativity (SI score $\leq 1$ ) of their expression in the tumor tissues (Fig. 4A and B). These data suggest that serum variables are more sensitive than the tissue counterparts. However, the change of the serum levels of HER2 was different between negative and positive groups. In patients with HER2-negative (SI score $\leq 1$ ), the serum level of HER2 was significantly decreased after tumor removal (M-AO vs. $\mathrm{M}-\mathrm{BO}$; $\mathrm{P}<0.05$ ), whereas in patients with HER2-positive (SI score $\geq 2$ ), no change of serum HER2 was observed after tumor removal ( $\mathrm{P}>0.05)$ (Fig. 4C).

Association of the concentrations of serum NAMPT, VEGF and HER2 with the clinicopathological features of breast cancer patients. Finally, the association of the serum levels of NAMPT, VEGF and HER2 with the clinicopathological features of breast cancer patients before and after tumor removal was examined. The serum levels of NAMPT as well as VEGF were significantly different in patients with breast cancer before and after operation and were found to be higher in patients before surgery $(\mathrm{P}<0.05)$ (Table IV), irrespective of the clinicopathological features. The serum level of HER2 was also higher in patients younger than 50 , with tumor size $\leq 2 \mathrm{~cm}$, without lymph node metastasis in clinical stage of 0-II before operation than those after operation $(\mathrm{P}<0.05)$. However in patients older than 50 , with tumor size $>2 \mathrm{~cm}$ and lymph node metastasis in clinical stage of III-IV, no significant difference of HER2 was observed before and after operation $(\mathrm{P}>0.05)$. By comparing with the clinicopathological features of patients, such as age ( $\leq 50$ vs. $>50)$, tumor size ( $\leq 2 \mathrm{vs} .>2 \mathrm{~cm})$, lymph node metastasis (yes vs. no), and clinical stage (0-II vs. III-IV), the serum levels of NAMPT, VEGF and HER2 were similar (all $\mathrm{P}>0.05$ ).

\section{Discussion}

The present study demonstrated that NAMPT, VEGF and HER2 were not only overexpressed in the tumor tissue, but also increased in the circulation in patients with breast cancer. To our knowledge this is the first report to propose a detection panel of the NAMPT/VEGF/HER2 triplet for the diagnosis as well as prognosis of human breast cancer.

It has been shown that the overexpression of NAMPT, VEGF, or HER2 is found in several carcinomas, including breast cancer (20-22). Previous studies showed that the levels of plasma NAMPT were higher in Chinese patients with breast cancer than in healthy controls (23), as well as that the levels of plasma VEGF were higher in premenopausal patients with early breast cancer than in normal premenopausal controls (16). It has also been reported that HER2 is a serum biomarker of breast cancer (24). However, these molecules are not examined together in the same patient with breast tumor (either benign or malignant). Importantly, the association of these variables with the clinicopathological characteristics of breast cancer has not been reported. In the present study we examined the individual as well as cumulative expression of these variables in the breast tissues from patients with tumors (benign or malignant) and also determined their serum levels. Furthermore, we analyzed the possible correlations between their expression in tissue and serum concentration before and after the tumor removal by surgery. To the best of our knowledge, current study is the first report to evaluate them as a biomarker-triplet for breast cancer diagnosis and also as an indicator of treatment effectiveness after tumor removal. 


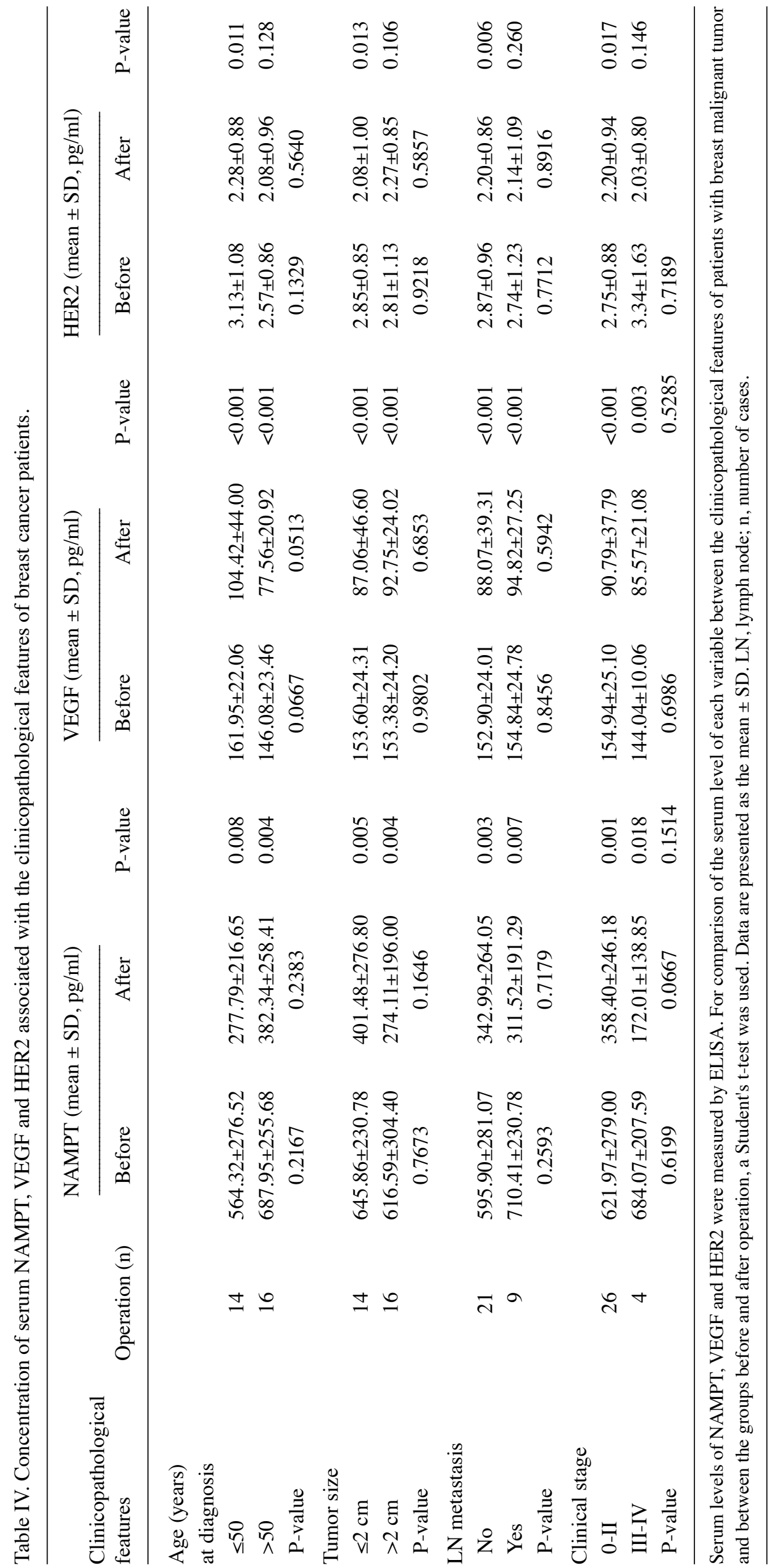


Our immunohistochemistry analysis showed that the expression of NAMPT, VEGF and HER2 was positive in most breast cancer tissue while they were negative in adjacent normal tissue, and their expression was higher in malignant tumors compared to the benign ones. Further analysis showed that the rate of IHC positivity for a single variable was $\sim 60 \%$ in malignant tumor, which was increased to $\sim 79 \%$ for two variables and to $90 \%$ for all three variables assessed together. These results imply a potential clinical applicability in molecular pathology for these variables to be used as a biomarker panel in the detection and prognosis of breast cancer. The change of the expression level from negative in healthy control to weak positive in benign tumor and to strong positive in malignant tumor suggests that the NAMPT/VEGF/HER2 triplet presents the ability to be a progression biomarker for tumorigenesis.

Compared with three variables in malignant tumors, NAMPT was the most sensitive marker showing $~ 6.64$-fold increase, followed by HER2 (2.52-fold) and VEGF (1.76-fold). The most important finding of the present study is that despite the differences in concentrations, essentially all three variables (NAMPT, VEGF and HER2) were found higher in the serum of breast cancer patients, indicating that the elevated NAMPT/VEGF/HER2 can be used as a diagnostic tool for human breast cancer. Furthermore, the decrease in the serum level of this biomarker-triplet after tumor removal suggests it to be a useful indicator of treatment efficacy and prognosis. In terms of the association of HER 2 expression, the serum level of HER2 detected by ELISA was significantly decreased after tumor removal in patients HER2-negative by ICH, but not in patients HER2-positive. These data suggest that in HER2-positive patients, NAMPT and VEGF rather than HER2 are most useful variables for monitoring the treatment effectiveness after surgery.

Increasing evidence demonstrates that NAMPT is a multifunctional enzyme which is important in metabolism and immune response as well as in cancer. It can affect metastatic activities and cell adhesive functions by regulating integrins in breast cancer (25). Overexpression of NAMPT is associated with aggressive pathological and molecular features, such as estrogen receptor negativity and HER2-enriched phenotypes (26), as well as malignancy and poor prognosis $(27,28)$. VEGF, an angiogenic marker, is found to be overexpressed in primary breast cancer (29) and plays a role in breast cancer angiogenesis (30), whereas HER 2 positivity and negativity are related to the therapy of breast cancer $(31,32)$. Previous study also showed that the overexpression of HER2 was significantly correlated to a higher expression of VEGF in breast cancer (21), but the clinical association of NAMPT/VEGF/HER2 with breast cancer has not been previously reported. The present study provides evidence of the relationship of these variables and their association with the clinicopathological features.

In summary, the present study demonstrated that NAMPT, VEGF and HER2 were overexpressed in breast tumors as well as elevated in the serum of patients with breast cancer and their circulating levels declined after tumor removal, suggesting a clinical application of this triplet as a biomarker for breast cancer diagnosis and as an indicator for treatment efficacy. The combined measurement of the triplet may improve the sensitivity of breast cancer diagnosis and potentially be used as a testing panel for the detection of malignant tumors, the assessment of treatment effectiveness, and the monitoring of the disease progression in patients with breast cancer. Thus, we propose that the biomarker triplet NAMPT/VEGF/HER2 can be used as a de novo detection panel for the diagnosis and prognosis of human breast cancer.

\section{Acknowledgements}

The present study was supported by grants from National Natural Science Foundation of China (81272880), the Shanghai Committee of Science and Technology (124119b1300) and Shanghai Municipal Health Bureau (2012-186) to G.X. and the Committee of Science and Technology of Jinshan (2013-3-15) to Y.Z. We thank Jimin Shi for pathological evaluation, and Jihong Zhang and Xiaoqun Lv for technique assistance.

\section{References}

1. Jemal A, Bray F, Center MM, Ferlay J, Ward E and Forman D: Global cancer statistics. CA Cancer J Clin 61: 69-90, 2011.

2. Fukuhara A, Matsuda M, Nishizawa M, Segawa K, Tanaka M, Kishimoto K, Matsuki Y, Murakami M, Ichisaka T, Murakami $\mathrm{H}$, et al: Visfatin: A protein secreted by visceral fat that mimics the effects of insulin. Science 307: 426-430, 2005.

3. Samal B, Sun Y, Stearns G, Xie C, Suggs S and McNiece I: Cloning and characterization of the cDNA encoding a novel human pre-B-cell colony-enhancing factor. Mol Cell Biol 14: 1431-1437, 1994.

4. Bi TQ, Che XM, Liao XH, Zhang DJ, Long HL, Li HJ and Zhao W: Overexpression of Nampt in gastric cancer and chemopotentiating effects of the Nampt inhibitor FK866 in combination with fluorouracil. Oncol Rep 26: 1251-1257, 2011.

5. Wang B, Hasan MK, Alvarado E, Yuan $\mathrm{H}, \mathrm{Wu} \mathrm{H}$ and Chen WY: NAMPT overexpression in prostate cancer and its contribution to tumor cell survival and stress response. Oncogene 30: 907-921, 2011.

6. Hufton SE, Moerkerk PT, Brandwijk R, de Bruïne AP, Arends JW and Hoogenboom HR: A profile of differentially expressed genes in primary colorectal cancer using suppression subtractive hybridization. FEBS Lett 463: 77-82, 1999.

7. Van Beijnum JR, Moerkerk PT, Gerbers AJ, De Bruïne AP, Arends JW, Hoogenboom HR and Hufton SE: Target validation for genomics using peptide-specific phage antibodies: A study of five gene products overexpressed in colorectal cancer. Int J Cancer 101: 118-127, 2002.

8. Shackelford RE, Bui MM, Coppola D and Hakam A: Over-expression of nicotinamide phosphoribosyltransferase in ovarian cancers. Int J Clin Exp Pathol 3: 522-527, 2010.

9. Folgueira MA, Carraro DM, Brentani H, Patrão DF, Barbosa EM, Netto MM, Caldeira JR, Katayama ML, Soares FA, Oliveira CT, et al: Gene expression profile associated with response to doxorubicin-based therapy in breast cancer. Clin Cancer Res 11: 7434-7443, 2005.

10. Shackelford R, Hirsh S, Henry K, Abdel-Mageed A, Kandil E and Coppola D: Nicotinamide phosphoribosyltransferase and SIRT3 expression are increased in well-differentiated thyroid carcinomas. Anticancer Res 33: 3047-3052, 2013.

11. Tian W, Zhu Y, Wang Y, Teng F, Zhang H, Liu G, Ma X, Sun D, Rohan T and Xue F: Visfatin, a potential biomarker and prognostic factor for endometrial cancer. Gynecol Oncol 129: 505-512, 2013.

12. Maldi E, Travelli C, Caldarelli A, Agazzone N, Cintura S, Galli U, Scatolini M, Ostano P, Miglino B, Chiorino G, et al: Nicotinamide phosphoribosyltransferase (NAMPT) is over-expressed in melanoma lesions. Pigment Cell Melanoma Res 26: 144-146, 2013.

13. Reddy PS, Umesh S, Thota B, Tandon A, Pandey P, Hegde AS, Balasubramaniam A, Chandramouli BA, Santosh V, Rao MR, et al: PBEF1/NAmPRTase/Visfatin: A potential malignant astrocytoma/glioblastoma serum marker with prognostic value. Cancer Biol Ther 7: 663-668, 2008. 
14. Dalamaga M, Archondakis S, Sotiropoulos G, Karmaniolas K, Pelekanos N, Papadavid E and Lekka A: Could serum visfatin be a potential biomarker for postmenopausal breast cancer? Maturitas 71: 301-308, 2012.

15. Thielemann A, Baszczuk A, Kopczyński Z, Kopczyński P and Grodecka-Gazdecka S: Clinical usefulness of assessing VEGF and soluble receptors sVEGFR-1 and sVEGFR-2 in women with breast cancer. Ann Agric Environ Med 20: 293-297, 2013.

16. Byrne GJ, McDowell G, Agarawal R, Sinha G, Kumar S and Bundred NJ: Serum vascular endothelial growth factor in breast cancer. Anticancer Res 27 (5B): 3481-3487, 2007.

17. Baskić D, Ristić P, Pavlović S and Arsenijević N: Serum HER2 and CA 15-3 in breast cancer patients. J BUON 9: 289-294, 2004.

18. Rocca A, Cancello G, Bagnardi V, Sandri MT, Torrisi R, Zorzino L, Viale G, Pietri E, Veronesi P, Dellapasqua S, et al: Perioperative serum VEGF and extracellular domains of EGFR and HER2 in early breast cancer. Anticancer Res 29: 5111-5119, 2009.

19. Wang X, Gui L, Zhang Y, Zhang J, Shi J and Xu G: Cystatin B is a progression marker of human epithelial ovarian tumors mediated by the TGF- $\beta$ signaling pathway. Int $\mathrm{J}$ Oncol 44: 1099-1106, 2014.

20. Dalamaga M, Karmaniolas K, Papadavid E, Pelekanos N, Sotiropoulos G and Lekka A: Elevated serum visfatin/nicotinamide phosphoribosyl-transferase levels are associated with risk of postmenopausal breast cancer independently from adiponectin, leptin, and anthropometric and metabolic parameters. Menopause 18: 1198-1204, 2011.

21. Linderholm B, Andersson J, Lindh B, Beckman L, Erlanson M, Edin K, Tavelin B, Grankvist K and Henriksson R: Overexpression of c-erbB-2 is related to a higher expression of vascular endothelial growth factor (VEGF) and constitutes an independent prognostic factor in primary node-positive breast cancer after adjuvant systemic treatment. Eur J Cancer 40: 33-42, 2004.

22. Kirkpatrick K, Ogunkolade W, Elkak A, Bustin S, Jenkins P, Ghilchik $M$ and Mokbel K: The mRNA expression of cyclo-oxygenase-2 (COX-2) and vascular endothelial growth factor (VEGF) in human breast cancer. Curr Med Res Opin 18: 237-241, 2002.

23. Li XY, Tang SH, Zhou XC, Ye YH, Xu XQ and Li RZ: Preoperative serum visfatin levels and prognosis of breast cancer among Chinese women. Peptides 51: 86-90, 2014.
24. Lam L, Czerniecki BJ, Fitzpatrick E, Xu S, Schuchter L, Xu X and Zhang $\mathrm{H}$ : Interference-free HER2 ECD as a serum biomarker in breast cancer. J Mol Biomark Diagn 4: 151, 2014.

25. Santidrian AF, LeBoeuf SE, Wold ED, Ritland M, Forsyth JS and Felding BH: Nicotinamide phosphoribosyltransferase can affect metastatic activity and cell adhesive functions by regulating integrins in breast cancer. DNA Repair (Amst) 23: 79-87, 2014.

26. Soncini D, Caffa I,Zoppoli G, Cea M, Cagnetta A, Passalacqua M, Mastracci L,Boero S, MontecuccoF, Sociali G, etal: Nicotinamide phosphoribosyltransferase promotes epithelial-to-mesenchymal transition as a soluble factor independent of its enzymatic activity. J Biol Chem 289: 34189-34204, 2014.

27. Lee YC, Yang YH, Su JH, Chang HL, Hou MF and Yuan SS: High visfatin expression in breast cancer tissue is associated with poor survival. Cancer Epidemiol Biomarkers Prev 20: 1892-1901, 2011.

28. Dalamaga M: Nicotinamide phosphoribosyl-transferase/visfatin: A missing link between overweight/obesity and postmenopausal breast cancer? Potential preventive and therapeutic perspectives and challenges. Med Hypotheses 79: 617-621, 2012.

29. Iovino F, Ferraraccio F, Orditura M, Antoniol G, Morgillo F, Cascone T, Diadema MR, Aurilio G, Santabarbara G, Ruggiero R, et al: Serum vascular endothelial growth factor (VEGF) levels correlate with tumor VEGF and p53 overexpression in endocrine positive primary breast cancer. Cancer Invest 26: 250-255, 2008 .

30. Lissoni P, Fugamalli E, Malugani F, Ardizzoia A, Secondino S, Tancini G and Gardani GS: Chemotherapy and angiogenesis in advanced cancer: Vascular endothelial growth factor (VEGF) decline as predictor of disease control during taxol therapy in metastatic breast cancer. Int J Biol Markers 15: 308-311, 2000.

31. Ross JS and Fletcher JA: The HER-2/neu oncogene in breast cancer: Prognostic factor, predictive factor, and target for therapy. Stem Cells 16: 413-428, 1998.

32. Park IH, Lee KS, Kang HS, Kim SW, Lee S, Jung SY, Kwon Y, Shin KH, Ko K, Nam BH, et al: A phase Ib study of preoperative lapatinib, paclitaxel, and gemcitabine combination therapy in women with HER2 positive early breast cancer. Invest New Drugs 30: 1972-1977, 2012. 